\title{
Analysis of Apple Inc.'s Innovation Dilemma From the Perspective of Leadership
}

\author{
Zixuan Liu ${ }^{1, *}$ \\ ${ }^{1}$ School of Economic and Management, Beijing Jiaotong University, Beijing, China \\ *Corresponding author. Email: 1450826320@qq.com
}

\begin{abstract}
Innovation plays the key role in the technology company. Currently, there are many studies exploring the model of company's innovation referring to the theoretical framework in the book named "The Dilemma of Innovators". However, there are no studies exploring it based on the perspective of leadership. This paper will analyze the innovation dilemma of Apple Inc. from the perspective of leadership and study it by comparing the differences between two leaders, Jobs and Cook. It's found that Jobs was more inclined to develop and promote the destructive technologies and Cook was more inclined to develop and promote the continuous technologies. The continuous technology can improve the performance of mature products based on the performance level that mainstream customers in major markets have valued. On the contrary, the destructive technology will lead to reducing the product performance and the products based on it are generally lower in price, simpler in performance, smaller in size. The different direction of their attention has directly led to the difference of Apple Inc. between these two eras. In order to avoid entering the dilemma of innovators and keep Apple Inc.'s leading position, Cook, the current CEO of Apple Inc., must take some certain measures. Experience shows that building an independent organization to face the new customer group directly is the best way to avoid entering the innovation dilemma.
\end{abstract}

Keywords: Leadership, Continuous technology, Disruptive technology, Value network, Innovator bonus.

\section{INTRODUCTION}

Apple Inc. has always stood at the forefront of industry technology relying on continuous innovative design. In a sense, Apple Inc. has surpassed the concept of products as a symbol of innovation. However, in recent years, the product has been criticized by the public and the media for its lack of innovation. Additionally, the iPhone just came on sale and it has frequently heard news of cold sales. It fell below the official website price within a few days of product listing. This paper will take this as a background to carry out research on Apple Inc.'s innovation model, and uses the theory of innovation dilemma as a framework to compare and analyze the characteristics of the two leaders from the perspective of leadership.

\section{THEORETICAL REVIEW}

The theory of Christensen's innovation dilemma is that management is the main reason which leads the leading companies to lose their first position. The decision will lead to business failure when leading companies are widely popular as the best companies in the world. The enterprise adopted the opinions of customers and actively invested in the research and development of new technologies. They carefully studied the market trend and systematically allocated investment capital to the innovative fields that brought the best rate of return. Eventually, they lost market leading position. On the contrary, it is right to invest in products with lower profit and poorer performance and make a big foray into small emerging markets, which is more likely to lead to success.

Introduce two concepts that is continuous technology and destructive technology. Most new technologies will improve the product performance. It is called continuous technology and all of them have one common that they improve the performance of mature products based on the performance level that mainstream customers in the major markets have valued. Destructive technology is that when it appears, at least in the short term, it will lead to reducing the product performance and making it lower than the 
have some

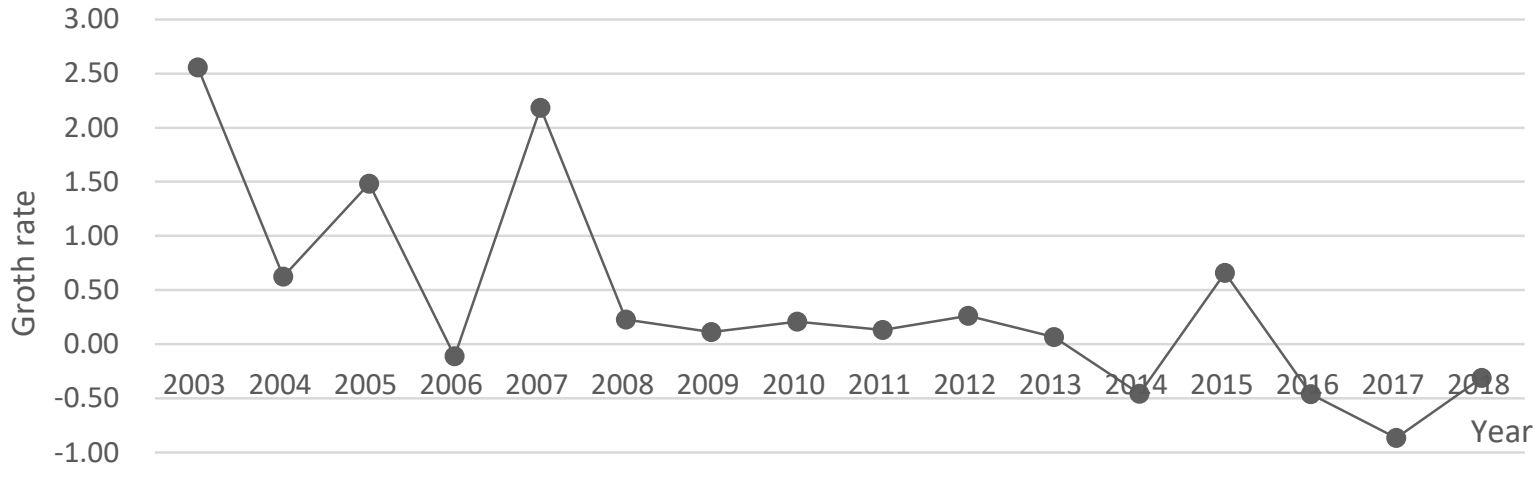

Figure 1 Apple Inc.'s patent application growth rate.

other characteristics valued by edge customers. Products based on destructive technology are generally lower in price, simpler in performance, smaller in size and often more customer friendly. Although these products have lower profit margins and will not bring greater profits. But, once they enter the mainstream market, they can launch a fierce attack on the previous technical products and change the product's competitive basis fundamentally form another mainstream market. As a result, companies that have been focusing on continuous technology will lose their leading position.

\section{CASE PROCESS ANALYSIS}

\subsection{Case Background}

Figure 1 is the growth rate of the number of patents applied by Apple Inc. in each year from 2003 to 2018. From 2003 to 2011, Jobs served as the CEO of Apple Inc., Cook took over from 2011 to the present. We can clearly see taking 2011 as the node. The average growth rate of the number of patent applications in the Cook era is significantly lower than that in the Jobs era.

\subsection{Case Study}

\subsubsection{Mature Companies Tend to Pay Attention to Continuous Technology and Fall into the Dilemma of Innovation}

Apple Inc., led by Steve Jobs, is an emerging company that has re-integrated innovative technologies into products and contributed to the industry's technological revolution. Emerging enterprises pay more attention to the development of destructive technologies or to reintegrate the destructive technologies that mature companies have abandoned. The impact of such destructive technologies is fatal and it is enough to change the entire industrial structure.
Now Apple Inc. seems to be a mature enterprise and not only has its own software and hardware development technology, systems, fixed products and services but also has a fixed market and customers. Therefore, Apple Inc. now pays more attention to the continuous technology than the Apple Inc. of the Jobs era. So it is easy to fall into the dilemma of innovation.

\subsubsection{Excessive Attention to Profit will Make the Company Lose its Leading Position.}

Pursuing profits and markets makes it easy for managers to do something meaningful to the business and ignore the development of destructive technologies. What makes sense is determined by the value network that the enterprise is in. The value network determines what products and services the enterprise must provide to solve the customer's problems and the cost the enterprise pays to solve these problems. However, the development of destructive technologies is more likely to occur in value networks with low gross profit margins and this area are ignored by managers easily. Cook is more focused on profits and markets than Jobs. Cook is better at operation and management and Jobs pays more attention to products and technology. Jobs once disagrees and disputes with the goals of the board due to the stubbornness and paranoia of the product, which eventually led to the expulsion of the board While Cook can dispose of the operational details such as the supply chain and cost to control to the extreme maximize profits. Fortune Magazine once commented that Cook is a very radical operator. Therefore, Cook pays more attention to continuous technology than Steve Jobs. So it is easy to fall into the dilemma of innovation. 


\subsubsection{Following the Market Will Ignore the Development of Disruptive Technologies}

Following the market is to improve product performance and allocate the resource system to the mainstream demand of the market according to the mainstream customers' major values. The products of destructive technology are lower in performance than the mature products of the mainstream market and the price is low. The market size is small and the profit margin is small so proposals to invest in destructive technologies are usually rejected, which can easily lead managers to miss the research and development and development of destructive technology products and thus easily lose the leading position. Jobs is the market leader and he is good at defining a market rather than participating in the snatching of existing markets. For example, his i-series products redefine consumer electronics products and make them "intelligent" and "toy" Road. He created products that can lead consumer demand and make them try and chase. Cook is following the market and he is used to listening to the opinions of the best customers to determine which products can get a large profit margin. As a result, the market commented that Apple Inc. has become more open and more modern than before. In summary, Cook, who is accustomed to following the market and more likely to focus on the continuous technology, $\mathrm{He}$ ignored the development of destructive technology and fall into the dilemma of innovation than Jobs.

\subsubsection{Over-satisfaction with Market Demands and the Push of Value Networks Make Them Face a Dilemma}

In the process of developing better products than competitors, in order to achieve higher selling prices and greater profit margins, suppliers often over-satisfy market demand and the products they provide to customers usually exceed the actual needs of customers or the actual price that the customer is ultimately willing to pay. However, when the supply of technology exceeds the requirements of mainstream customers or the performance improvement that can be digested, customers will no longer make better choices based on product performance but based on the evolution from functionality to reliability and then to convenience, and finally to the price selection to select products. Now Apple Inc.'s products such as mobile phones, tablets or laptops, they are suffering from fierce impact from competitors in the same industry. The important reason is that the products provided to customers exceed the actual needs of customers or customers are willing to pay actual price.

The power of the value network can push enterprises to develop upwards into a new value network that is different from the previous ones but this unusually powerful influence will prevent companies from moving to the lower level market. The rational model of moving to the high level market has an important strategic impact but it also creates a competitive vacuum for the low level value network. It attracts new companies whose technology and cost structure more closely match this value network to participate in the competition. Now, Apple Inc. seems to have trapped itself in the value network of high level consumers and its initial advantages has also led it to reluctant to lay down profits to seize the low level market, which made Apple Inc. fell into the dilemma of innovation.

\section{COUNTERMEASURES AND SUGGESTIONS}

\subsection{Become a Leader in Disruptive Technological Change}

Leading in continuous technology does not bring many advantages but a lot of evidence shows that leading disruptive technology is very important. When disruptive technologies emerge, companies that first enter emerging markets will win huge returns and establish clear first-mover advantages. As for enterprises, the risk of creating new markets is much lower than that of entering a competitive and mature market but the rate of return is much higher, and as the enterprise continues to grow and grow it is becoming more and more difficult to enter emerging markets as early as possible. Therefore, in order to obtain a first-mover advantage, Apple Inc. must take certain measures to create a market to develop destructive technology products actively.

\subsection{Adopt a Learning and Discovery Plan instead of an Execution Plan}

The market application field of destructive technology is not only unknown but also unknowable. Therefore, the strategies and plans adopted by managers in response to destructive technology changes should be related to discovery and learning rather than execution. Because it is impossible to find destructive technology projects to succeed at once, it is important to learn to discover and retain resources so that new business projects can have sufficient resources to support when they find the right direction later. In the process of finding a market for destructive technologies, we must learn to not only use the resources of mainstream institutions to deal with disruptive changes but also avoid the values and processes of mainstream institutions. 


\subsection{Create an Independent Institution to Deal with the Development of Small Markets}

Because the markets spawned by disruptive technologies were relatively small in the beginning and the orders received by companies in these markets were also small orders, in order to cope with the small market cannot solve the problem of the short-term growth demand of large institutions, it need create a small independent agency to develop and promote disruptive technological innovation projects. Find an organization that matches the size of the target market to develop destructive technology projects. The customer base of this organization also needs this technology. The needs of customers can improve the profitability of the enterprise so that innovation can get the resources needed and it is easy to be satisfied with small opportunities and small gains.

\subsection{Maintain Excellent Management Skills}

When some excellent enterprises pursue profit and growth rate, their managers have used the best management skills and led to the failure of the enterprise. But companies should not give up the capabilities and decision-making processes that once made them shine in the mainstream market because these management skills are unable to deal with the threats posed by destructive technologies. Because most of the innovation challenges faced are essentially continuous innovation. The original intention of companies cultivating these capabilities is to deal with such innovation challenges. The managers only need to realize that these capabilities, cultures and methods have value only under certain conditions.

\section{CONCLUSION}

Christensen's theory of innovation dilemma aims to point out why excellent management skills are the main factors that make companies lose their leading position. This paper compares the leadership differences between two leaders of Jobs and Cook and concludes that Apple Inc. is facing the dilemma of innovators. The study concluded that Jobs, who valued products and technology, paid more attention to the development of destructive technologies, while Cook, who focused on profits and markets, paid more attention to the development of continuous technologies. Paying attention to the continuous technology and ignoring the development of destructive technology is more likely to lead to the failure of leading companies. Both the former film king Kodak and the once brilliant Nokia have proved this. In order to escape from the dilemma of innovation, Apple Inc. can develop a learning and discovery plan and create an independent organization that matches the size of the target customer group. Developing destructive technology to become a leader in destructive technology and take the lead to deal with the threat of innovation dilemma. As an exploratory case study, this research is just the beginning and related research needs to be continuously in-depth.

\section{REFERENCES}

[1] Chen Ping, Leng Yuanhong. A Comparative Study on Innovation Issues of High-Tech Enterprises_— Taking Apple Inc. and Samsung as Examples[J]. Technology Economics and Management Research,2012(5):50-54.

[2] Gu Chen, Xu Shanshan, The Enlightenment of Apple Inc.'s Business Model to Chinese Enterprises in the Transition Period[J].Jiangsu Commercial Theory,2012(4):134-136.

[3] Ding Xiaohui, The most important class before graduation from Harvard Business School [J].Eastern Entrepreneur, 2013(11):43-43.

[4] Li Xiaomei [Compilation].What will happen after entrepreneurs leave [J].International Advertising, 2011(10):124-125.

[5] Guo Li, Wang Dongzhi, Zheng Xiaoxiao. The New Development of Leadership Style Theory and the Improvement of Modern Enterprise Leadership_-Taking Jobs as an Example [J].Shang, 2014(10):31-32.

[6] Shao Xinyao. The decisive role of leaders in corporate culture: Taking the development of Apple Inc. as an example [J]. Chinese and foreign entrepreneurs, 2012(8):132-133.

[7] Zhang Meng. Seeing leadership through Steve Jobs' Apple Inc.[J]. Science Education Journal, 2012(17):103-103.

[8] Christensen, Clayton M, Leslie, Don. The Innovator's Dilemma $[\mathrm{M}] / / \quad$ The innovator's dilemma: Harvard Business School Press, 1997:382. 